\title{
CORRESPONDENCE \\ Radial Flow of Compressible Fluids Between Discs
}

\section{Correspondence from N. A. KOLOKOL'TSOV (Institute of Atomic Energy, Moscow)}

I have read with pleasure the article by Tsung Yen $\mathrm{Na}$ published in the August 1965 issue of the Aeronautical Quarterly. The results, especially those concerning the critical diameter and the choking phenomena, are very interesting.

It seems though that at least part of the results could be given in a simpler form and thus be made clearer by replacing Mach number $M$ by the reduced velocity $\lambda=u / a \star$, where $u$ is the radial velocity ( $V$ in the author's notation) and $a \star$ the critical velocity of sound. The two variables are bound together by the equation

$$
\lambda^{2}=\frac{\frac{x+1}{2} M^{2}}{1+\frac{x-1}{2} M^{2}}
$$

The author's equation (8) may then be written in the form

$$
\frac{d X}{d \lambda}=-\frac{X}{\lambda} \frac{1-\lambda^{2}}{1-\frac{x-1}{x+1} \lambda^{2}-\frac{x}{x+1} X \lambda^{2}}
$$

Equation $\left(8^{\prime}\right)$ is of the same type as $(8)$ and can be solved only numerically. We find no simplification here. The integral curves $\lambda(X, C)$ of $\left(8^{\prime}\right)$ will be analogous in shape to the curves $M(X, C)$ in the author's Fig. 2. One can calculate the curves $\lambda(X, C)$ by using the curves in Fig. 2 and equation (1) of this Note.

After that we find the velocity from the simple formula

$$
u=\lambda a \star
$$

and the pressure and density (see, for instance, Ref. 1 of this Note) from

$$
\begin{aligned}
& p=\frac{x+1}{2 x} \frac{G}{g A} \frac{1-\frac{x-1}{x+1} \lambda^{2}}{\lambda}, \\
& \rho=\frac{G}{g A \lambda a^{\star}},
\end{aligned}
$$

where $G$ is the constant mass flow through the area $A$, which is known from boundary conditions.

It is felt that the possibility of using simple formulae $\left(9^{\prime}\right),\left(10^{\prime}\right),\left(12^{\prime}\right)$ instead of the numerical integration of differential equations (9) to (13) makes the analysis easier. The equations of the limiting curves (17) and (18), corresponding to the change of sign in the derivatives $d p / d X$ and $d \rho / d X$, may be easily derived from $\left(9^{\prime}\right)$ and $\left(10^{\prime}\right)$, first 


\section{CORRESPONDENCE}

differentiating by $\lambda$, then eliminating $d \lambda / d X$ from $\left(8^{\prime}\right)$ and equating the corresponding expressions to zero. For instance equation (17) will be written in the form

$$
2\left(1-\frac{x-1}{x+1} \lambda^{2}\right)=X\left(1+\frac{x-1}{x+1} \lambda^{2}\right) .
$$

The observations made seem to indicate that the use of $\lambda$ instead of $M$ leads to simpler results. This may help if the method suggested by Tsung Yen $\mathrm{Na}$ is used for analogous problems.

\section{Reference}

1. Galperin, V. G., Millionstchikov, M. D., Simonov, L. A. and Khristianovich, S. A. Prikladnaya gasovaya dinamica. USSR, 1947.

\section{Reply from TSUNG YEN NA (University of Michigan)}

The author appreciates deeply the comments by Mr. Kolokol'tsov and agrees that the introduction of the reduced velocity $\lambda=u / a^{\star}$ makes certain parts of the analysis easier, although the conclusions remain the same.

Based on the introduction of the reduced velocity, $\lambda$, and replacing the crosssectional area, $A$, by $2 \pi r h$, equations $\left(8^{\prime}\right),\left(12^{\prime}\right),(10)$ and $\left(9^{\prime}\right)$ in Mr. Kolokol'tsov's comment become

$$
\begin{gathered}
\frac{d X}{d \lambda}=-\frac{X}{\lambda} \frac{1-\lambda^{2}}{1-\frac{k-1}{k+1} \lambda^{2}-\frac{k}{k+1} X \lambda^{2}}, \\
V=\lambda a \star \\
\rho=\frac{f G}{X \pi h^{2} \lambda a^{\star}} \\
p=\frac{f G}{X \pi h^{2}} \frac{1-\frac{k-1}{k+1} \lambda^{2}}{\lambda} \frac{k+1}{2 k} a \star,
\end{gathered}
$$

where $f, G$ and $h$ are the friction factor (assumed constant), the mass flow rate and the distance between the discs respectively. $a^{\star}$ can be computed from the equation

$$
a \star=\sqrt{\left(\frac{2 k}{k+1} R T_{0}\right)}
$$

For a given inlet condition, namely, $X_{i}, \lambda_{i}, V_{i}, \rho_{i}, p_{i}$, the relation between $X$ and $\lambda$ can only be solved numerically, as shown in both the paper and the discussion. For given $\rho_{i}$ and $p_{i}$, the temperature at the inlet is fixed by the ideal gas law, which in turn can be used to compute $a \star$. To get solutions to $V, \rho$ and $p$ at any $X$, we replace equations (2), (3) and (4) by the following equations. 